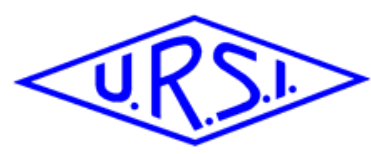

\title{
A wireless method for drone identification and monitoring using AIS technology
}

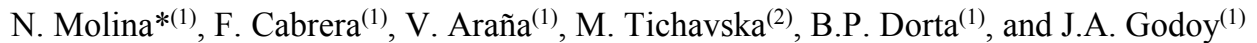 \\ (1) University of Las Palmas de Gran Canaria, Las Palmas, Spain, e-mail: nicolas.molina@ulpgc.es; \\ (2) Exmile Solutions Limited (MarineTraffic), London, UK; e-mail: milusetichavska@marinetraffic.com
}

\begin{abstract}
The fast growth of UAV (Unmanned Aerial Vehicle) technology in the last years has allowed to extend the use of these devices in many applications. However, the massive use of drones has alerted many governments about an inadequate usage, mainly in terms of security and terrorism. In regards to this problem, some laws about drone usage have been approved by some countries. Among these laws, it is mandatory that all drones are identified and monitored all the time. In this paper, a wireless prototype to identify drones is proposed. To that end, the AIS (Automatic Identification System) is used to transmit parameters as name, ID, speed or the course of drones. This proposed solution can solve the drone identification problem and even, allows to monitor this device in real time. A prototype of this method has been implemented and tested in a real environment, around many locations in the island of Gran Canaria.
\end{abstract}

\section{Introduction}

The use of UAVs (Unmanned Aerial Vehicles), commonly known as drones, plays an important role in our society. Originally, these devices were used in military environments, but nowadays are used for commercial, researching and entertainment applications [1]. Although all activities carried out for the users of these devices are regulated by the laws of each country, the increasing use of drones has raised awareness in governments of many countries about an inadequate usage of these, mainly in terms of security and terrorism [2].

In regards to this problem, restrictive laws about drone usage have not been approved yet in Europe and USA. However, there is a significant interest in stablishing new laws that regulate the available flight zones for drones, the maximum cargo which can be conveyed in these devices and even, the need to identify and monitor a drone all the time. The active observation of drones is currently limited to subjective and qualitative human sense impressions. This, in addition to obstacles for human sight in regular flight conditions outlines a major challenge towards drone identification and monitoring.

In this paper, a wireless prototype for drone identification is proposed. For this purpose, the marine communication system AIS (Automatic Identification System) has been used. The AIS, is an automatic tracking system used on ships and by vessel traffic services since 2003. Its communication protocol allows the transmission of static, dynamic and voyage information, including the name, ID, speed or course of the vessel [3]. This system operates over two VHF frequencies (191.975 and $192.025 \mathrm{MHz}$ ), uses a TDMA scheme to avoid collisions between stations and can reach a coverage of more than 100 NM [4]. Any vessel that transmits AIS messages must be registered with a unique number named MMSI (Maritime Mobile Service Identity), assigned by a national maritime authority, and the NMEA (National Marine Electronics Association) protocol is used to provide an AIS message format [5].

In conclusion, the AIS can be effectively used as wireless alternative for drone identification and monitoring. Not only it allows near real time tracking but may also incorporate parameters related to flight metrics and sensor value connected to the system may be also incorporated.

\section{Prototype architecture}

The wireless prototype proposed is integrated in an amateur drone. This prototype is formed by four blocks:

- Flight unit: it is formed by the drone's structure (3D Robotics Y6) and a flight control device (Pixhawk).

- Control unit: it is based on Raspberry Pi, which is used as a microcontroller, and can generate the NMEA format for new AIS messages.

- $\quad$ RF unit: in addition to an AIS transceiver (SRT Cobalt), that is capable to transmit and receive AIS messages, it has a VHF and GPS antennas.

- Supply unit: it is used a DC-DC converter which is connected to a LiPo battery to provide power enough for the prototype.

In Figure 1, a block diagram of the proposed prototype is shown.

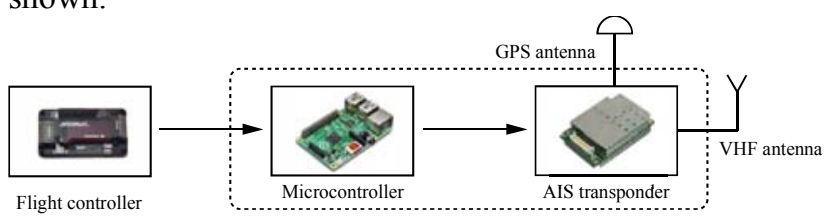

Figure 1. Prototype's block diagram 


\section{Drone identification and monitoring}

An AIS transceiver can transmit all static parameters of a drone (name, MMSI, vendor ID, type of vessel), but even many dynamic parameters such as speed, course or location. However, there are some parameters which cannot be registered (due to the AIS protocol in place) but are very important for drone monitoring. These relate to the angular information (pitch, roll and yaw) or height. To solve this problem, and to enable the use of a custom AIS message executed from the microcontroller, an algorithm has been designed. With this algorithm, it is possible to capture a drone value and then generate a NMEA message format by means of a HEHDT command, that is used to provide the header true data. The steps of this algorithm are shown in Figure 2.

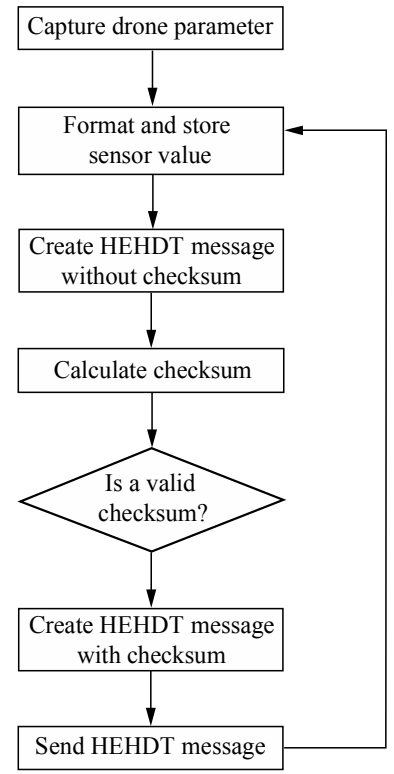

Figure 2. Algorithm to send drone parameters with AIS message format

\section{Measurements and results}

To test the prototype in a real environment, different locations around the island of Gran Canaria have been selected (Figure 3). These locations include coastal and mountain zones which could affect the signal propagation.

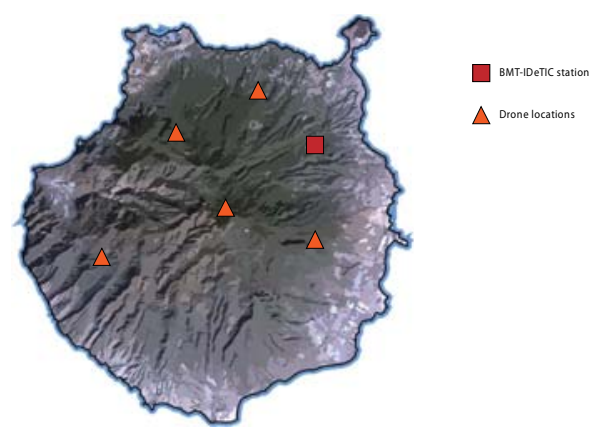

Figure 3. Measurement locations in Gran Canaria
In these places, drone height parameters have been transmitted through the prototype. These measurements are shown in Table 1.

Table 1. Height measurements around Gran Canaria

\begin{tabular}{|l|l|}
\hline Location & Drone height \\
\hline La Isleta & $4 \mathrm{~m}$ \\
\hline Arucas & $3.5 \mathrm{~m}$ \\
\hline Artenara & $4.2 \mathrm{~m}$ \\
\hline Teror & $2.1 \mathrm{~m}$ \\
\hline
\end{tabular}

\section{Conclusions}

A wireless method for drone identification and monitoring based on the AIS has been proposed and tested. Results obtained in the island of Gran Canaria and the implemented prototype are made available to identify and monitor a drone in coastal or mountain environments. Moreover, additional drone information (height) has been transmitted through this prototype.

\section{Acknowledgements}

The authors acknowledge the work carried out by Juan Domingo Santana Urbín for his enormous contribution during this research. Also the support and continued assessment by MarineTraffic. This work was supported by the Spanish Government under Grant TEC201460283-C3-2-R Project and the University of Las Palmas de Gran Canaria ULPGC2015-01 Project.

\section{References}

1. Lords, H. O. Civilian Use of Drones in the EU.

2. Hartmann, K., \& Giles, K. (2016, May). UAV exploitation: A new domain for cyber power. In $C y b e r$ Conflict (CyCon), 2016 8th International Conference on(pp. 205-221). IEEE.

3. Cabrera, F., Molina, N., Tichavska, M., \& Arana, V. (2016). Automatic Identification System modular receiver for academic purposes. Radio Science, 51(7), 1038-1047. 4. Lessing, P. A., Bernard, L. J., Tetreault, B. J., \& Chaffin, J. N. (2006, September). Use of the Automatic Identification System (AIS) on autonomous weather buoys for maritime domain awareness applications. In OCEANS 2006(pp. 1-6). IEEE.

5. Uribe, J. D. J. R., Guzman, D. C., \& Bermudez, C. (2015, May). Methodology to calculate the coverage of AIS station implementing base station traffic measurements. In Communications and Computing (COLCOM), 2015 IEEE Colombian Conference on (pp. 1-6). IEEE. 